\title{
Albert Division Algebras in Characteristic three contain Cyclic Cubic Subfields.
}

\author{
Holger P. Petersson \\ Fachbereich Mathematik \\ FernUniversität - Gesamthochschule in Hagen \\ D - 58084 Hagen \\ Germany \\ Helmut Röhrl zum siebzigsten Geburtstag gewidmet
}

\begin{abstract}
It is shown that a finite-dimensional absolutely simple nonsingular Jordan division algebra of degree 3 over a field containing the third roots of unity admits a cyclic cubic subfield.
\end{abstract}

The question as to whether every Albert division algebra contains a cyclic cubic subfield, whose significance derives from its connection with twisted compositions in the sense of Springer [2,6], has been raised by Albert [1] and remains unsolved to this day. In the presence of the third roots of unity, an affirmative answer has been given by Petersson-Racine [4] provided the underlying base field has characteristic different from 2 and 3 . It is the purpose of the present note to remove this extra hypothesis by establishing the following result.

Theorem. Let $k$ be a field containing the third roots of unity. Then every Albert division algebra over $k$ contains a cyclic cubic subfield.

The hypothesis on $k$ being automatic if the characteristic is 3 , the statement of the title drops out as an immediate corollary.

We whish to prove the theorem in the more general setting of absolutely simple nonsingular Jordan algebras of degree 3 and begin by summarizing the relevant facts needed for that purpose; the reader ist referred to McCrimmon [3] for details. Let $k$ be an arbitrary field and $\mathcal{J}$ a finite-dimensional unital quadratic Jordan algebra of degree 3 oder $k$. Then $\mathcal{J}$ arises form a cubic form $N: \mathcal{J} \rightarrow k$ (given by its norm) with adjoint $\sharp: \mathcal{J} \rightarrow \mathcal{J}$ (given by the 
numerator of the inversion map) and base point $1 \in \mathcal{J}$ (given by the identity element). We assume throughout that $\mathcal{J}$ is absolutely simple (hence remains simple under all base field extensions) and nonsingular, i.e., the trace form

$$
T=-\left(D^{2} \log N\right)(1): \mathcal{J} \times \mathcal{J} \longrightarrow k
$$

has this property (which follows from absolute simplicity unless we are in characteristic 2). Every element $x$ in any scalar extension of $\mathcal{J}$ satisfies the generic minimum polynomial

$$
\lambda^{3}-T(x) \lambda^{2}+S(x) \lambda-N(x)
$$

where $T(x)=T(x, 1)$ and $S(x)=T\left(x^{\sharp}\right)$. The quadratic form $S$ bilinearizes to

$$
\begin{aligned}
S(x, y) & =S(x+y)-S(x)-S(y) \\
& =T(x) T(y)-T(x, y) .
\end{aligned}
$$

We write $S^{0}$ for the restriction of $S$ to $\mathcal{J}^{0}=\operatorname{ker} T$. If char $k \neq 3, S^{0}$ is nonsingular. If char $k=3, \operatorname{rad} S^{0}=k 1$.

Now suppose $\mathcal{J}$ is reduced and hence, for some composition algebra $C$ over $k$ and some diagonal matrix $g \in \mathrm{GL}_{3}(k)$, identifies with the Jordan algebra $\mathrm{H}_{3}(C, g)$ of 3 -by-3 $g$-hermitian matrices with entries in $C$ and scalars down the diagonal. We write $e_{i}(1 \leq i \leq 3)$ for the absolutely primitive standard idempotents of $\mathcal{J}$ and observe

$$
\sum e_{i}=1, e_{i}^{\sharp}=0, T\left(e_{i}, e_{j}\right)=\delta_{i j} \quad(1 \leq i, j \leq 3) .
$$

Hence $u_{i}=e_{i}-e_{3} \in \mathcal{J}^{0}(i=1,2)$, and (2), (3) imply

$$
S^{0}\left(u_{i}\right)=S^{0}\left(u_{1}, u_{2}\right)=-1 \quad(i=1,2) .
$$

Proposition 1. Assume $k$ has characteristic not 3 and contains the third roots of unity. Then the quadratic form $S^{0}$ is isotropic.

Proof. By Springer's Theorem on quadratic forms [5, Chap. 2, 5.3] (where the restriction to fields of characteristic not two is easily seen to be unnecessary), 
we may assume that $\mathcal{J}$ is reduced. Then, letting $\zeta \in k$ be a primitive third root of unity, (4) implies

$$
S^{0}\left(u_{1}+\zeta u_{2}\right)=-\left(1+\zeta+\zeta^{2}\right)=0 .
$$

Proposition 2. Assume char $k=3$. Then the quadratic form $S^{0}$ represents the element $-1 \in k$.

Proof. Applying Springer's Theorem again, this time to the quadratic form $q \perp<1>$, where $q$ is induced by $S^{0}$ on $\mathcal{J}^{0} / k 1$, we may again assume that $\mathcal{J}$ is reduced. But then (4) again immediately yields what we want.

We are now ready for the extended version of our original theorem promised earlier.

Theorem 3. Let $k$ be a field containing the third roots of unity and let $\mathcal{J}$ be a finite-dimensional absolutely simple nonsingular Jordan division algebra of degree 3 over $k$. Then $\mathcal{J}$ contains a cyclic cubic subfield.

Proof. For char $k \neq 3$, it suffices to exhibit a Kummer extension of degree 3 in $\mathcal{J}$. By (1) the existence of such an extension is equivalent to $S^{0}$ being isotropic, and Proposition 1 completes the proof. For char $k=3$, the cubic subfield of $\mathcal{J}$ generated by a nonzero element $u \in \mathcal{J}^{0}$ because of (1) has the discriminant

$$
d=-4 S^{0}(u)^{3}-27 N(u)^{2}=-S^{0}(u)^{3} .
$$

Hence the existence of cyclic cubic subfields of $\mathcal{J}$ is equivalent to $S^{0}$ representing $-1 \in k$, and Proposition 2 completes the proof.

Without the hypothesis on $k$, Theorem 3 is known to be false in dimension 9 (see Petersson-Racine [4, Proposition 5] for a counter example), the case of dimension 27 (Albert algebras) in characteristic not three still being open. 


\section{References}

1. Albert, A. A. On exceptional Jordan division algebras. Pacific J. Math. $\mathbf{1 5}$ (2) (1965), 377-404.

2. Knus, M.-A., A. S. Merkurjev, M. Rost, J.-P. Tignol. "The book on Involutions". To appear.

3. McCrimmon, K. The Freudenthal-Springer-Tits constructions of exceptional Jordan algebras. Trans. Amer. Math. Soc. 139 (1969), 495-510.

4. Petersson, H. P., M. L. Racine. Cubic subfields of exceptional simple Jordan algebras. Proc. Amer. Math. Soc. 91 (1) (1984), 31-36.

5. Scharlau, W. "Quadratic and hermitian forms". Grundlehren Math. Wiss. Bd. 270. Berlin, Heidelberg, New York, Tokyo: Springer 1985.

6. Springer, T. A. "Oktaven, Jordan-Algebren und Ausnahmegruppen". University of Göttingen Lecture Notes: Göttingen, 1963. 